\title{
Supplemental Information for: Creation and Release of an Updated NIST DART-MS Forensics Database
}

\author{
Edward Sisco ${ }^{\text {a* }}$, Arun S. Moorthy ${ }^{\mathrm{a}}$, Laura M. Watt ${ }^{\mathrm{a}}$ \\ aNational Institute of Standards and Technology, 100 Bureau Drive, Gaithersburg, MD 20899 \\ *DARTdata@nist.gov, 301-975-2093
}

\section{NIST DART-MS Database Builder}

The NIST DART-MS Database Builder program was designed to evaluate DART-MS mass spectra and collate a database. This program was written using $R$ 3.6.2 $2^{1}$. External R-packages used in the program include: data.table ${ }^{2}$, readxl $^{3}$, OrgMassSpecR $^{4}$ and enviPat ${ }^{5}$. Specific application of functions from each external package will be described as required. In addition, Open Babel ${ }^{6,7}$ functionality was used to convert between chemical structure formats.

The data necessary to construct a DART-MS Database, here-in referred to as a DB, must be provided as a master file and a main folder. The master file should be an excel worksheet (.xlsx extension), describing the entries to be contained in the final DB, including metadata about the compounds (e.g., molecular formula, SMILES) and other relevant comments about the data collection process. The main folder should consist of several subdirectories, each containing DART-MS mass spectra as txt files ${ }^{8}$ obtained at the named orifice energy. The standard/expected subdirectories are $+30 \mathrm{~V},+60 \mathrm{~V}$, and $+90 \mathrm{~V}$. There should be one spectrum in each orifice energy subdirectory for every database entry listed in the master file.

\section{Automated database building and evaluation protocol}

Step 1. Initiate Database. A DB is initiated by reading in the master file excel sheet using the read_excel function from the readxl package and stored as a data.table object from the data.table package.

\footnotetext{
${ }^{1}$ R Core Team (2019). R: A language and environment for statistical computing. R Foundation for Statistical Computing, Vienna, Austria. URL https://www.R-project.org/.

${ }^{2}$ Matt Dowle and Arun Srinivasan (2019). data.table: Extension of 'data.frame'. R package version

1.12.8. https://CRAN.R-project.org/package $=$ data.table

${ }^{3}$ Hadley Wickham and Jennifer Bryan (2019). readxl: Read Excel Files. R package version 1.3.1.

https://CRAN.R-project.org/package=readxl

${ }^{4}$ Nathan Dodder (2017). OrgMassSpecR: Organic Mass Spectrometry. R package version 0.5-3. https://CRAN.R-project.org/package=OrgMassSpecR

${ }^{5}$ Loos, M., Gerber, C., Corona, F., Hollender, J., Singer, H. (2015). Accelerated isotope fine structure calculation using pruned transition trees, Analytical Chemistry 87(11), 5738-5744.

6 http://openbabel.org/wiki/Main Page

${ }^{7}$ O'Boyle, N. M., Banck, M., James, C. A., Morley, C., Vandermeersch, T., \& Hutchison, G. R. (2011). Open Babel: An open chemical toolbox. Journal of cheminformatics, 3(1), 33.

8 The present implementation also accepts spectra in jsp file type. Other file types may be considered in future implementations.
} 
Step 2. Confirm sufficient data is available. The spectra in each "energy" subdirectory of the main folder should correspond to the compounds listed in the master file and vice versa. If there is an inconsistency, the program will halt.

Step 3. Generate structure information from SMILES (assumed to be correct) and mass values from molecular formulas (assumed to be correct) for all compounds in the master file. Structure conversions are done through system calls to Open Babel. Up to three open babel commands are run per compound in the master file: (1) a conversion of SMILES to an SDF that explicitly includes hydrogen atoms, (2) a conversion of SMILES to an SDF that excludes hydrogen atoms and, if necessary, (3) a conversion from SMILES to InChI Key. Generating monoisotopic mass values for each compound was done using a modified version of the MonoisotopicMass function from the OrgMassSpecR package. The function was modified to compute masses for compounds that included silicon, iodine, sodium, and potassium atoms.

Step 4. Collect mass spectra from folders and evaluate (1) mass calibration and (2) dimer presence, in the lowest energy spectra. Compounds where the molecular ion is greater than $\pm 0.005 \mathrm{Da}$ from its expected $\mathrm{m} / \mathrm{z}$ value are marked for revision. Spectra which contained a peak within $0.2 \mathrm{Da}$ of the projected dimer peak, and with intensity greater than $30 \%$ of the intensity of the protonated molecule, are marked for re-measurement.

Step 5. Check for fragmentation consistency across mass spectra measured at multiple orifice energy values for the same analyte. Fragmentation should increase with orifice energy. Fragmentation in a mass spectrum is approximated through a weighted average $\mathrm{m} / \mathrm{z}$ value. Lower weighted average $\mathrm{m} / \mathrm{z}$ values indicate higher fragmentation. Compounds for which fragmentation is deemed inconsistent across orifice energies are marked for revision.

Step 6. Annotate peaks and evaluate noise in each mass spectrum. Given a compounds structure as a Mol object, the program computes a list of all possible fragment formulas derived through breaking individual bonds in the compound. This is accomplished by selecting a row from the connection block of the Mol object and designating each numbered atom as the starting atoms of two fragments. By reading through the entire connection block and identifying how the other atoms are connected to the designated starting atoms, two fragments can be generated. Note that if the bond broken is within a ring structure, the resulting formulas of both computed fragments will be identical to the original molecule. Multiple loops through the connection block may be necessary to create complete fragments. The mass values, assuming all isotopic variants, of all unique fragments are computed using the isopattern function from the enviPat package. Possible adduct mass values are also computed. Given the list of mass values, each peak in the mass spectrum is annotated with a possible structure when a mass is matched within $\pm 0.005 \mathrm{Da}$. Peaks that cannot be explained by the list of fragments and subsequent mass values are deemed potential noise. A noise fraction is computed as the intensity of potential noise peaks to the total intensity of all peaks. Spectra for which the computed noise fraction was greater than 0.45 are marked for revision.

Step 7. Print. Structure and spectral information for each compound at each orifice energy are printed as a single SDF file. 


\section{Software Availability}

Base R (https://www.r-project.org/) and Open Babel (https://openbabel.org/wiki/Main_Page) are freely available and the version appropriate for the users operating system should be installed prior to running the NIST DART-MS Database Builder (DBB) program. Source code for the NIST DART-MS DBB is available for download at https://github.com/asm3-nist/DART-MS-DBB. The NIST Lib2NIST tool for converting SDF files into NIST format libraries is available for download at https://chemdata.nist.gov.

\section{Compounds in the Database}

Compounds in the initial updated database, broken down into classes. The database will be updated over time and therefore this list may not be complete. Compounds with a shaded blue box indicate those whose spectra are identical to the original database.

\begin{tabular}{|c|c|c|}
\hline \multicolumn{3}{|c|}{ Amphetamines } \\
\hline 2-Fluoromethamphetamine & Benzphetamine & Methylephedrine \\
\hline 3,4-Methylenedioxyamphetamine & Chlorphentermine & Methylphenidate \\
\hline $\begin{array}{c}3,4- \\
\text { Methylenedioxyethylamphetamine }\end{array}$ & Dexmethylphenidate & $\begin{array}{c}\text { N-benzyl-3,4- } \\
\text { Dimethoxyamphetamine }\end{array}$ \\
\hline $\begin{array}{c}3,4- \\
\text { Methylenedioxymethamphetamine }\end{array}$ & DOC & $\mathrm{N}$-Ethylamphetamine \\
\hline 3-Fluoromethamphetamine & Ephedrine & Phendimetrazine \\
\hline 4-Fluoroamphetamine & Fenfluramine & Phentermine \\
\hline 4-MAPB & Levetiracetam & Phenylepherine \\
\hline $5-$ IT & Lisdexamfetamine & Phenylpropanolamine \\
\hline 5-MMPA & Mephentermine & p-Methoxyamphetamine \\
\hline $6-I T$ & Methamphetamine & Pseudoephedrine \\
\hline Amphetamine & $\begin{array}{l}\text { Methamphetamine Methyl } \\
\text { Carbamate }\end{array}$ & \\
\hline \multicolumn{3}{|c|}{ Antidepressants } \\
\hline Benactyzine & Doxepin & Nefazodone \\
\hline Bupropion & Fluoxetine & Nortriptyline \\
\hline Clomipramine & Imipramine & Paroxetine \\
\hline Desipramine & Mirtazapine & Prpotriptyline \\
\hline \multicolumn{3}{|c|}{ Antipsychotics } \\
\hline Butaperazine & Loxapine & Promazine \\
\hline Chlorpromazine & Molindone & Quetiapine \\
\hline Clozapine & Olanzapine & \\
\hline Haloperidol & Prochloroperazine & \\
\hline \multicolumn{3}{|c|}{ Arylcyclohexamines } \\
\hline 2-Fluoro Deschloroketamine & 3-Methoxy PCP & Ketamine \\
\hline 3-Chloro-PCP & 4-Methoxy-PCP & Methoxetamine \\
\hline 3-Fluoro Deschloroketamine & 4-methyl-PCP & Phencyclidine \\
\hline 3-fluoro-PCP & BTCP & \\
\hline 3-Hydroxy-PCP & Deschloroketamine & \\
\hline \multicolumn{3}{|c|}{ Barbiturates } \\
\hline Amobarbital & Hexobarbital & Pentobarbital \\
\hline Butabarbital & Metharbital & Secobarbital \\
\hline \multicolumn{3}{|c|}{ Benzodiazepines } \\
\hline Bromazepam & Etizolam & Nitrazepam \\
\hline Chlordiazepoxide & Flualprazolam & Nordiazepam \\
\hline Clobazam & Flubromazolam & Oxazepam \\
\hline Clonazepam & Flunitrazepam & Temazepam \\
\hline Clonazolam & Flurazepam & Triazolam \\
\hline
\end{tabular}




\begin{tabular}{|c|c|c|}
\hline Diazepam & Lorazepam & Zolazepam \\
\hline Diclazepam & Medazepam & a-hydroxy Alprazolam \\
\hline Escitalopram & Metizolam & \\
\hline Estazolam & Midazolam & \\
\hline \multicolumn{3}{|c|}{ Cannabinoids (Natural) } \\
\hline Cannabichromene & Cannabinol & $\Delta$-9-Tetrahydrocannabinol \\
\hline Cannabidiol & $\Delta 6 a(10 a)$-Tetrahydrocannabinol & \\
\hline Cannabidiolic Acid Methyl Ester & $\Delta$-8-Tetrahydrocannabinol & \\
\hline \multicolumn{3}{|c|}{ Cathinones } \\
\hline 2,3-Dimethylmethcathinone & 4-Ethylmethcathinone & Methylone \\
\hline 2-Ethylmethcathinone & $\begin{array}{l}\text { 4-fluoro-3-methyl- } \alpha- \\
\text { Pyrrolidinovalerophenone }\end{array}$ & MPHP \\
\hline 2-Fluoromethcathinone & 4-Fluoro- $\alpha$-PHP & N,N-Dimethylpentylone \\
\hline 2-Methylmethcathinone & $\begin{array}{c}\text { 4-methyl-a- } \\
\text { Pyrrolidinoisohexanophenone }\end{array}$ & Naphyrone \\
\hline $\begin{array}{l}\text { 3,4-dichloro-N-cyclohexyl } \\
\text { Methcathinone }\end{array}$ & 4-Methylbuphedrone & N-Ethyl Heptedrone \\
\hline 3,4-Dimethylmethcathinone & 4-Methyl-N,N-dimethylcathinone & N-Ethyl Hexedrone \\
\hline $\begin{array}{c}\text { 3,4-Methylenedioxy-N- } \\
\text { benzylcathinone }\end{array}$ & 5-APB & N-Ethylbuphedrone \\
\hline $\begin{array}{l}\text { 3',4'-Methylenedioxy-N-tert- } \\
\text { butylcathinone }\end{array}$ & 5-APDB & N-Ethylcathinone \\
\hline 3,4-Methylenedioxypyrovalerone & 5-MAPB & N-ethylhexedrone \\
\hline 3-Bromomethcathinone & 5-MAPDB & N-Ethyl-N-methylcathinone \\
\hline 3-Chloro-N,N-dimethylcathinone & 6-APDB & Pentedrone \\
\hline 3-Ethylmethcathinone & 6-MAPB & Pentylone \\
\hline 3-Fluoromethcathinone & Butylone & $\alpha-P B P$ \\
\hline 3-fluoro-a-Pyrrolidinohexanophenone & Cathine & a-Propylaminopentiophenone \\
\hline 3-Methylbuphedrone & Diethylpropion & $\alpha-P V P$ \\
\hline 3-Methylmethcathinone & Flephedrone & a-Pyrrolidinohexanophenone \\
\hline $\begin{array}{c}\text { 4-bromo- } \alpha- \\
\text { Methylaminovalerophenone }\end{array}$ & Mephedrone & a-Pyrrolidinoisohexanophenone \\
\hline 4-Chloroethcathinone & MePPP & \\
\hline 4-Chloro-N,N-Dimethylcathinone & Methedrone & \\
\hline \multicolumn{3}{|c|}{ Cocaines \& Tropanes } \\
\hline Cocaine & & \\
\hline \multicolumn{3}{|c|}{ Fentanyls } \\
\hline $\begin{array}{l}\text { 2,2,3,3-Tetramethyl-Cyclopropyl } \\
\text { fentanyl }\end{array}$ & $\mathrm{N}-(2 \mathrm{C}-\mathrm{B}-$ fly) Fentanyl & para-Chloroisobutyryl fentanyl \\
\hline 2,3-Seco-Fentanyl & $\mathrm{N}-(2 \mathrm{C}-\mathrm{C})$ Fentanyl & para-Fluoro Acrylfentanyl \\
\hline 2',5'-dimethoxy Fentanyl & $\mathrm{N}-(2 \mathrm{C}-\mathrm{D})$ Fentanyl & para-Fluoro Crotonyl fentanyl \\
\hline 2'-Fluoro ortho-Fluorofentanyl & $\mathrm{N}-(2 \mathrm{C}-\mathrm{E})$ Fentanyl & para-Fluoro Cyclopentyl fentanyl \\
\hline 2-Furanyl fentanyl & $\mathrm{N}-(2 \mathrm{C}-\mathrm{G})$ Fentanyl & para-Fluoro Cyclopropyl fentanyl \\
\hline 2'-methyl Acetyl fentanyl & $\mathrm{N}-(2 \mathrm{C}-\mathrm{I})$ Fentanyl & para-Fluoro Furanyl fentanyl \\
\hline 2'-Methyl Fentanyl & $\mathrm{N}-(2 \mathrm{C}-\mathrm{iP})$ Fentanyl & $\begin{array}{l}\text { para-Fluoro Furanyl fentanyl 3- } \\
\text { furancarboxamide isomer }\end{array}$ \\
\hline $\begin{array}{c}\text { 3-Furanyl fentanyl 3- } \\
\text { furancarboxamide isomer }\end{array}$ & $\mathrm{N}-(2 \mathrm{C}-\mathrm{N})$ Fentanyl & para-Fluoro Methoxyacetyl fentanyl \\
\hline 3'-methyl Acetyl fentanyl & $\mathrm{N}-(2 \mathrm{C}-\mathrm{P})$ Fentanyl & $\begin{array}{l}\text { para-Fluoro Tetrahydrofuran } \\
\text { fentanyl }\end{array}$ \\
\hline 3'-Methyl Fentanyl & $\mathrm{N}-(2 \mathrm{C}-\mathrm{T})$ Fentanyl & para-Fluoro Valeryl fentanyl \\
\hline $\begin{array}{l}\text { 4'-Fluoro, para-fluoro-trans-3-methyl } \\
\text { Fentanyl }\end{array}$ & $\mathrm{N}-(2 \mathrm{C}-\mathrm{T}-2)$ Fentanyl & para-Fluoroacetyl fentanyl \\
\hline 4'-Fluorofentanyl & N-(2C-T-4) Fentanyl & para-Fluorobutyryl fentanyl \\
\hline 4'-methyl Acetyl Fentanyl & N-(2C-T-7) Fentanyl & para-Fluorofentanyl \\
\hline 4'-Methylfentanyl & N-(2C-TFM) Fentanyl & para-Fluoroisobutyryl fentanyl \\
\hline 4-Phenyl Fentanyl & $\mathrm{N}-(3,4,5-\mathrm{TMA})$ Fentanyl & para-methoxy Acetyl fentanyl \\
\hline Acetyl fentanyl & N-(3C-B-fly) Fentanyl & para-Methoxy Acrylfentanyl \\
\hline
\end{tabular}




\begin{tabular}{|c|c|c|}
\hline Acetyl Norfentanyl & $\mathrm{N}$-(3-ethylindole) Norfentanyl & para-Methoxy Furanyl fentanyl \\
\hline Acrylfentanyl & N-(6-APB) Fentanyl & $\begin{array}{l}\text { para-Methoxy Methoxyacetyl } \\
\text { fentanyl }\end{array}$ \\
\hline Alfentanil & N-(6-APDB) Fentanyl & $\begin{array}{l}\text { para-methoxy Tetrahydrofuran } \\
\text { fentanyl }\end{array}$ \\
\hline Benzodioxole fentanyl & $\mathrm{N}$-(DOB) Fentanyl & para-Methoxy Valeryl fentanyl \\
\hline Benzyl Acrylfentanyl & $\mathrm{N}-(\mathrm{DOBU})$ Fentanyl & para-Methoxy-Butyrylfentanyl \\
\hline Benzyl Carfentanil & $\mathrm{N}-(\mathrm{DOC})$ Fentanyl & para-Methoxyfentanyl \\
\hline Benzylfentanyl & $\mathrm{N}$-(DOET) Fentanyl & para-Methyl Acetyl fentanyl \\
\hline Butyryl fentanyl & $\mathrm{N}$-(DOI) Fentanyl & para-methyl Acrylfentanyl \\
\hline Butyryl norfentanyl & $\mathrm{N}$-(DOM) Fentanyl & para-methyl Butyryl fentanyl \\
\hline Carfentanil & $\mathrm{N}$-(MDA) Fentanyl & para-Methyl Cyclopentyl fentanyl \\
\hline cis-3-methyl Butyryl fentanyl & $\mathrm{N}$-(phentermine) Fentanyl & para-Methyl Cyclopropyl fentanyl \\
\hline cis-3-methyl Fentanyl & $\begin{array}{l}\text { N,N-Dimethylamido-despropionyl } \\
\text { fentanyl }\end{array}$ & para-Methyl Furanyl fentanyl \\
\hline cis-3-methyl Norfentanyl & N-benzyl Furanyl norfentanyl & para-methyl Isobutyryl fentanyl \\
\hline cis-3-methyl Thiofentanyl & $\begin{array}{c}\text { N-benzyl para-fluoro Cyclopropyl } \\
\text { norfentanyl }\end{array}$ & para-methyl Methoxyacetyl fentanyl \\
\hline cis-Isofentanyl & N-Benzyl para-fluoro Norfentanyl & $\begin{array}{l}\text { para-methyl Tetrahydrofuran } \\
\text { fentanyl }\end{array}$ \\
\hline Crotonyl fentanyl & N-Benzyl phenyl norfentanyl & para-Methylfentanyl \\
\hline Cyclobutyl fentanyl & N-methyl Cyclopropyl norfentanyl & para-Toluoyl fentanyl \\
\hline Cyclohexyl fentanyl & $\mathrm{N}$-methyl Norcarfentanil & Phenoxyacetyl fentanyl \\
\hline Cyclopentenyl fentanyl & $\mathrm{N}$-methyl Norfentanyl & Phenyl fentanyl \\
\hline Cyclopentyl fentanyl & Norcarfentanil & Phenylacetyl fentanyl \\
\hline Cyclopropyl Fentanyl & Norfentanyl & Pivaloyl fentanyl \\
\hline $\begin{array}{l}\text { Despropionyl 2'-fluoro ortho- } \\
\text { Fluorofentanyl }\end{array}$ & Norsufentanil & Remifentanil \\
\hline Despropionyl meta-Methylfentanyl & Ocfentanil & Remifentanil Acid \\
\hline Despropionyl para-Fluorofentanyl & ortho-Fluoro Acrylfentanyl & Senecioylfentanyl \\
\hline Ethoxyacetyl fentanyl & ortho-Fluoro Furanyl fentanyl & Sufentanil \\
\hline Fentanyl & ortho-Fluorofentanyl & Tetrahydrofuran fentanyl \\
\hline Fentanyl Carbamate & ortho-Fluoroisobutyryl fentanyl & $\begin{array}{c}\text { Tetrahydrofuran fentanyl 3- } \\
\text { tetrahydrofurancarboxamide isomer }\end{array}$ \\
\hline Fentanyl Methyl Carbamate & ortho-isopropyl Furanyl fentanyl & Tetrahydrothiophene fentanyl \\
\hline Furanyl norfentanyl & ortho-methoxy Furanyl Fentanyl & Thienyl fentanyl \\
\hline Heptanoyl fentanyl & ortho-Methoxy-Butyryl fentanyl & Thiofentanyl \\
\hline Hexanoyl fentanyl & ortho-Methyl Acetyl fentanyl & Thiophene Fentanyl \\
\hline Isobutyryl fentanyl & ortho-Methyl Acrylfentanyl & trans-3-methyl Fentanyl \\
\hline Isovaleryl fentanyl & ortho-Methyl Cyclopropyl fentanyl & trans-3-Methyl Thiofentanyl \\
\hline meta-Fluoro Furanyl fentanyl & ortho-methyl Furanyl Fentanyl & Valeryl fentanyl \\
\hline meta-Fluoro Methoxyacetyl fentanyl & $\begin{array}{l}\text { ortho-Methyl Methoxyacetyl } \\
\text { fentanyl }\end{array}$ & $\alpha^{\prime}$-methoxy Fentanyl \\
\hline meta-Fluorobutyryl fentanyl & ortho-methyl Phenyl fentanyl & a-methyl Acetyl Fentanyl \\
\hline meta-Fluorofentanyl & ortho-Methylfentanyl & a'-Methyl Butyryl fentanyl \\
\hline meta-Fluoroisobutyryl fentanyl & para-Bromofentanyl & a-Methyl Butyryl fentanyl \\
\hline meta-Methyl Acetyl fentanyl & para-Chloro Acrylfentanyl & a-methyl Thiofentanyl \\
\hline meta-Methyl Cyclopropyl fentanyl & para-chloro Cyclobutyl Fentanyl & a-Methylfentanyl \\
\hline meta-Methyl Furanyl fentanyl & para-Chloro Cyclopentyl fentanyl & $\beta$-Hydroxyfentanyl \\
\hline meta-Methyl Methoxyacetyl fentanyl & para-Chloro Cyclopropyl fentanyl & $\beta$-Hydroxythioacetyl fentanyl \\
\hline meta-Methylfentanyl & para-Chloro Furanyl fentanyl & $\beta^{\prime}$-Hydroxythiofentanyl \\
\hline Methoxyacetyl fentanyl & $\begin{array}{l}\text { para-Chloro Methoxyacetyl } \\
\text { fentanyl }\end{array}$ & $\beta$-methyl Acetyl Fentanyl \\
\hline $\mathrm{N}$-(2,5-DMA) Fentanyl & para-Chloro Valeryl fentanyl & $\beta$-Methyl Fentanyl \\
\hline $\mathrm{N}$-(2-APB) Fentanyl & para-Chlorobutyryl fentanyl & $\beta^{\prime}-$ Phenyl fentanyl \\
\hline N-(2C-B) Fentanyl & para-Chlorofentanyl & \\
\hline \multicolumn{3}{|c|}{ Local Anesthetics } \\
\hline Butacaine & Dibucaine & \\
\hline Butamben & Tetracaine & \\
\hline
\end{tabular}




\begin{tabular}{|c|c|c|}
\hline \multicolumn{3}{|c|}{ Lysergamides } \\
\hline LSD & $\begin{array}{l}\text { Lysergic acid N- } \\
\text { (methylpropyl)amide }\end{array}$ & \\
\hline \multicolumn{3}{|c|}{ Noortropics } \\
\hline 4-Fluoro Phenibut & N-methyl-4,4-difluoro Modafinil & \\
\hline \multicolumn{3}{|c|}{ NSAIDs } \\
\hline Aminopyrine & Ibuprofen & Oxyphenbutazone \\
\hline Antipyrine & Ketoprofen & Piroxicam \\
\hline Celecoxib & Ketorolac & Salicylamide \\
\hline Etodolac & Meclofenamate & \\
\hline Flurbiprofen & Naproxen & \\
\hline \multicolumn{3}{|c|}{ Opiates } \\
\hline Buprenorphine & Heterocodeine & O-6-Monoacetylmorphine \\
\hline Codeine & Hydrocodone & Oxycodone \\
\hline Desomorphine & Hydromorphone & Oxymorphone \\
\hline Heroin & Morphine & Pseudocodeine \\
\hline \multicolumn{3}{|c|}{ Opioids (non-Fentanyl) } \\
\hline 2-fluoro MT-45 & Flunitazene & para-methyl AP-237 \\
\hline 2-methyl AP-237 & Isopropyl U-47700 & Pentazocine \\
\hline 3,4-Ethylenedioxy U-47700 & Isotodesnitazene & Propyl U-47700 \\
\hline 3,4-Ethylenedioxy U-51754 & Isotonitazene & Salvinorin $\mathrm{A}$ \\
\hline 3,4-Methylenedioxy U-47700 & levo-Propoxyphene & Tapentadol \\
\hline 4-phenyl U-51754 & Loperamide & Tramadol \\
\hline AH 7921 & Meperidine & $U-47700$ \\
\hline AP-238 & Methadone & U-47931E \\
\hline Brorphine & Methoxy U-47700 & U-48520 \\
\hline Butorphanol & Metodesnitazene & U-48753E \\
\hline Cyclopropyl U-47700 & Metonitazene & U-48800 \\
\hline Dextromethorphan & MT-45 & U-49900 \\
\hline Dextrorphan & $\mathrm{N}, \mathrm{N}$-didesmethyl U-47700 & U-50488 \\
\hline Diphenoxylate & Naloxone & U-51754 \\
\hline d-Propoxyphene & N-Desmethyl U-47700 & UF-17 \\
\hline Etodesnitazene & N-methyl U-47931E & W-18 \\
\hline \multicolumn{3}{|c|}{ Others } \\
\hline Brucine & Ecrgocalciferol & Nicotine \\
\hline Cinchonine & Epinephrine & Pindolol \\
\hline Cotinine & Ibutamoren Mesylate & Quinine \\
\hline Coumarin & Melatonin & Theobromine \\
\hline Dimethylsulfone & Neopine & $\alpha$-Tocopherol Acetate \\
\hline \multicolumn{3}{|c|}{ Pharmaceuticals (Other) } \\
\hline Albendazole & Cyclobenzaprine & Methoxsalen \\
\hline Albuterol & Diethylcarbamazine & Methylclothiazide \\
\hline Amantadine & Diphenylpyraline & Methyldopa \\
\hline Anastrozole & Donepezil & Methyprylon \\
\hline Arterenol Bitartrate & Exemestane & Metixene \\
\hline Aspirin & Fencamfamine & Metoprolol \\
\hline Atenolol & Fluticasone Propionate & Nadolol \\
\hline Atomoxetine & Gabapentin & Nitazoxanide \\
\hline Atropine & Gemfibrozil & Ondansetron \\
\hline Betamethasone & Griseofulvin & Papaverine \\
\hline Bisacodyl & Guaifenesin & Pentoxifylline \\
\hline Bisoprolol & Indinavir & Pentylenetetrazol \\
\hline Bithionol & Isotretinoin & Phenyltoloxamine \\
\hline Brompheniramine & Ketoconazole & Pilocarpine \\
\hline Carbromal & Lamotrigine & Pregabalin \\
\hline Cephalexin & Levamisole & Pyrilamine \\
\hline Cetirizine & Loratadine & Pyrimethamine \\
\hline
\end{tabular}




\begin{tabular}{|c|c|c|}
\hline Chlorcyclizine & Mazindol & Quinidine \\
\hline Chlorophenamine & MDAI & Sildenafil \\
\hline Chlorothalidone & Meclizine & Tadalafil \\
\hline Chloroxazone & Mephenesin & Warfarin \\
\hline Cimetidine & Mesoridazine & Xylazine \\
\hline Clopidogrel & Metaproterenol & Zolpidem \\
\hline Cyclizine & pyrilene & \\
\hline \multicolumn{3}{|c|}{ Phenethylamines } \\
\hline 25B-NBOH & $2 \mathrm{C}-\mathrm{C}$ & Mescaline \\
\hline 25B-NBOMe & $2 \mathrm{C}-\mathrm{D}$ & Mescaline NBOMe \\
\hline 25C-NBOMe & $2 \mathrm{C}-\mathrm{H}$ & Sibutramine \\
\hline $25 \mathrm{I}-\mathrm{NBOH}$ & $2 \mathrm{C}-\mathrm{I}$ & $\beta$-hydroxy 2C-B \\
\hline 25I-NBOMe & $2 \mathrm{C}-\mathrm{T}-2$ & Mescaline \\
\hline $2 \mathrm{C}-\mathrm{B}$ & 3,4-Dimethoxyphenethylamine & \\
\hline 25B-NBOH & $2 \mathrm{C}-\mathrm{C}$ & \\
\hline \multicolumn{3}{|c|}{ Piperazines } \\
\hline 1-(3-Chlorophenyl)piperazine & 1-(4-Methoxyphenyl)piperazine & 1-Benzylpiperazine \\
\hline \multicolumn{3}{|c|}{ Precursors } \\
\hline 2-Fluorophenyl Cyclopentyl Ketone & MMDPPA & para-fluoro 4-ANPP \\
\hline 4-Anilino-N-phenethylpiperidine & N-Moc-MDMA & PCC \\
\hline \multicolumn{3}{|c|}{ Steroids } \\
\hline 4-Androsten-3,6,17-trione & Dromostanolone Enanthate & Methyltrienolone \\
\hline 4-Androstenedione & Dromostanolone Propionate & Nandrolone Laurate \\
\hline Androsta-1,4-dien-3,11,17-trione & Drostanolone & Nandrolone Phenylpropionate \\
\hline Androstanolone valerate & Epitestosterone & Oxymesterone \\
\hline Androsterone & Estradiol Enanthate & Stanolone \\
\hline Bolandione & Estrone & Stanozolol \\
\hline Boldenone & Ethinyl estradiol & Testosterone \\
\hline Boldenone Undecylenate & Hydrocortisone & Testosterone Acetate \\
\hline Clostebol & Mesterolone & Testosterone Decanoate \\
\hline Cortisone Acetate & Mestranol & Testosterone Enanthate \\
\hline Dehydroisoandrosterone & Methandriol & Testosterone Isocaproate \\
\hline Dienestrol & Methandrostenolone & Testosterone Phenylpropionate \\
\hline Diethylstilbestrol & Methenolone Enanthate & Testosterone propionate \\
\hline Dihydroxyprogesterone Acetophenide & Methyltestosterone & \\
\hline \multicolumn{3}{|c|}{ Synthetic Cannabinoids } \\
\hline 4-cyano ADB-BUTINACA & AM2233 & JWH-250 \\
\hline 4-Cyano-CUMYL-BUTINACA & AM694 & JWH-302 \\
\hline 4-Fluoro MDMB-BUTINACA & Benzyl-4-cyano BUTINACA & JWH-398 \\
\hline 5-Chloro-AKB48 & CP 47,497 & MAM2201 \\
\hline 5-Cl-UR144 & CP 47,497-C8-homolog & MDMB-CHMICA \\
\hline 5F-AKB48 & CP 55,940 & MMB-CHM7AICA \\
\hline 5-fluoro MMB-P7AICA & CUMYL-CBMINACA & MPP-PICA \\
\hline 5Fluoro-AB-PINACA & EAM2201 & NM2201 \\
\hline 5-Fluoro-AKB48 & FDU-PB-22 & PX 2 \\
\hline 5-Fluoro-AMB & FUB-144 & RCS-4 \\
\hline 5-Fluoro-PB-22 & FUB-AKB48 & RCS-8 \\
\hline A-796,260 & $\mathrm{HU}-210$ & STS-135 \\
\hline AB-CHMINACA & $\mathrm{HU}-211$ & THJ \\
\hline AB-FUBINACA isomer 5 & iPDMB-FUBINACA & THJ2201 \\
\hline AB-PINACA & JWH-015 & UR-144 \\
\hline ADB-CHMINACA & JWH-073 & URB754 \\
\hline ADB-FUBINACA & JWH-122 & XLR11 \\
\hline ADB-PINACA & JWH-122 N-(4-pentenyl) analog & XLR11 N-(2-fluoropentyl) isomer \\
\hline AKB48 & JWH-200 & XLR11 N-(3-fluoropentyl) isomer \\
\hline AM1220 & JWH-201 & XLR11 N-(4-fluoropentyl) isomer \\
\hline AM2201 & JWH-203 & \\
\hline AM2201 benzimidazole analog & JWH-210 & \\
\hline
\end{tabular}




\begin{tabular}{|c|c|c|}
\hline \multicolumn{2}{|c|}{ Tryptamines } \\
\hline 4-Acetoxy-N,N-diethyltryptamine & $\begin{array}{c}\text { 5-Methoxy-N,N- } \\
\text { methylisopropyltryptamine }\end{array}$ & N,N-Diisopropyltryptamine \\
\hline 4-Hydroxy-MIPT & Acetylpsilocin & N,N-Dipropyltryptamine \\
\hline 5-Methoxy DALT & Bufotenine & N,N-Methylisopropyltryptamine \\
\hline 5-Methoxy-N,N-diethyltryptamine & Diethyltryptamine & N-Methyltryptamine \\
\hline 5-Methoxy-N,N-Diisopropyltryptamine & Dimethyltryptamine & Psilocin \\
\hline 5-Methoxy-N,N-Dimethyltryptamine & Hordenine & \\
\hline
\end{tabular}

